\title{
Current Insights on Cholangiocarcinoma Research: a Brief Review
}

\author{
Vivek Bhakta Mathema, Kesara Na-Bangchang*
}

\begin{abstract}
Colangiocarcinoma (CCA) is a progressively fatal disease which generally occurs due to malignant transformation of hepatic biliary cholangiocytes. The incidence of CCA has been increasing worldwide and there is an urgent requirement for effective diagnosis and treatment strategies against this devastating disease. Different factors including liver-fluke infestation, viral hepatitis, exogenous nitrosamine-mediated DNAdamage, and chronic inflammation have been linked to CCA genesis. However, the risk factors and underlying complex mechanisms leading to development of CCA are not sufficiently understood to devise an effective targeted treatment therapy. In this review, we summarize currently known epidemiological and pathological aspects of the disease and briefly describe various potential biomarkers and experimental anticancer phytochemicals related to CCA research. In addition, we also sum up recent findings that link chronic inflammation of hepatic biliary cholangiocytes with CCA. The collective information concisely presented in this article would provide useful insights into the current understanding of this cancer.
\end{abstract}

Keywords: Cholangiocarcinoma - biomarkers - Opisthorchis viverrini - inflammation

Asian Pac J Cancer Prev, 16 (4), 1307-1313

\section{Introduction}

Cholangiocarcinoma (CCA) basically refers to the cancer arising from malignant transformation of cholangiocytes, the epithelial cells lining the hepatic biliary tree. The CCA accounts for almost $15 \%$ of all hepatobiliary malignancies and are difficult to diagnose with dismal prognosis. The disease is progressively fatal and less than $5 \%$ of patients suffering from advanced stages of CCA survive for up to five years (Oh et al., 2005; Ustundag and Bayraktar, 2008). CCA is lethal unless the malignancy is diagnosed at its early stages and surgically removed. However, lack of single effective early diagnostic procedure and complex nature of the disease presents a great challenge for timely detection and treatment of CCA (Blechacz et al., 2011). Recent years have seen a surge in research related to the CCAassociated inflammation and immunological aspects of the disease to identify and correlate specific host immune response against CCA (Razumilava and Gores, 2012). In addition, the increasing incidents of CCA and absence of proper prophylaxis strategy has led to an urgent demand for identification and development of biomarkers that might hold clinical potential for timely diagnosis of the disease (Ruys et al., 2013).

In this review, we briefly discuss about the epidemiological and immunological aspects of CCA and the disease-associated pathogens. Moreover, we also summarize current trends in biomarker and anticancer research associated with diagnosis and potential therapeutic intervention of this life-threatening disease.

\section{Epidemiology and Classification of Cholangiocarcinoma}

CCA is comparatively rare form of carcinoma and constitutes less than $1 \%$ of overall human malignancies. Nonetheless, the disease is reportedly a leading form of liver-associated cancer after hetaocellular carcinoma (HCC) in several continents including parts of Europe and America (Parkin et al., 1993; Ustundag and Bayraktar, 2008). In USA alone, the incidence of CCA is 1-2 cases per 100,000 which equates to 3,500 new cases each year (Anderson et al., 2004). In disease incidence is also high is parts of Asia including northeastern Thailand and Laos (Sripa et al., 2009). Separate study indicates that the affected CCA cases are relatively more in males on their 60s-70s as compared to the females of similar age group (Shaib and El-Serag, 2004). However, it is questionable that weather observed prevalence of CCA is merely due to actual increase in CCA incidence or better diagnostic options available today. The CCA can be anatomically categorized as perihilar CCA (pCCA), distal CCA, and intrahepatic CCA (iCCA) depending upon the site of malignancy (Marrero, 2014). The pCCAs can arise from anywhere between the second-order biliary ducts and 
region above the site of cystic duct origin. The dCCA can develop in regions between the cystic duct origin and the hepatopancreatic ampulla. The iCCA originates from intrahepatic biliary tract which spread throughout the tract and can also form intrahepatic lesions. The pCCA and dCCA are sometimes collectively represented as extrahepatic CCA (Blechacz et al., 2011; Razumilava and Gores, 2012). Clinical statistics suggests that the iCCA-associated incidence and mortality rate is on the rise as compared to the perihilar and distal CCA (Shaib and El-Serag, 2004; Khan et al., 2012; Yao et al., 2014). Even though specific etiology behind the CCA remains unclear, the factors including congenital fibropolycystic liver disease, intrahepatic biliary stones, cirrhosis, liver fluke infestation, and viral Hepatitis B and C are considered as predisposing conditions for CCA. In particular, the primary sclerosing cholangitis (PSC) is considered as one of the most common risk factors of CCA with lifetime prevalancy for CCA reaching up to $10 \%$ among PSC patients (Burak et al., 2004). Similarly, chronic exposure to toxins, nitrosamines and dioxin are also considered as significant risk factors for CCA (Tyson and El-Serag, 2011; Razumilava and Gores, 2012). Thus, understanding the role of multiple risk factors and their possible interaction along with patient's clinical history is essential for CCA research.

\section{Cholangiocarcinoma and Inflammation}

Chronic inflammation has been attributed to initiate, assist or induce tumor growth in several diseases which in turn can presumably result in cancer (Takeuchi and Akira, 2010). Aberrant production of interleukin (IL)-6, a pro-inflammatory cytokine, has been correlated with the predisposition of chronic biliary tract inflammation to CCA (Johnson et al., 2012). Although the actual mechanism is barely understood, it seemingly implicates the role of IL- 6 for promoter methylation and expression of growth regulator genes that potentially contribute to carcinogenesis (Wehbe et al., 2006). In particular, the involvement of IL- 6 in tumor progression has been associated with modulation of expression of selected microRNAs which are responsible for post-transcriptional regulation of messenger RNA (mRNA) involved in synthesis of DNA methyltransferase, a crucial enzyme required for DNA methylation (Fabbri et al., 2007; Braconi et al., 2010). Moreover, IL-6 was attributed to promote survival of malignant cholangiocyte xenografts via an autocrine-paracrine mechanism in athymic mice (Braconi et al., 2010). Evidences from few investigation also suggest that during certain helminthic infection, the host immune system can exhibit IL-6 and metastasis-associated protein 1 (MAP-1)-regulated chronic inflammatory response that may contribute to development of CCA (Sripa et al., 2009; Nair et al., 2011). Likely, in parts of Thailand where CCA incidence is more frequent, the IL-6 receptor polymorphism has been frequently observed in Opisthorchiasis-linked CAA patients (Prayong et al., 2014).

The Nuclear factor kappa-B (NF-kB) is a major transcription factor associated with a wide range of cellular responses including inflammation, cell survival, and proliferation (Takeuchi and Akira, 2010). Results from in vitro studies conducted in CCA cell line suggested that diethyldithiocarbamate, a major metabolite of disulfiram, suppressed the metastatic activity of the cancer cells via inhibiting NF-kB pathway (Srikoon et al., 2013). Since this transcription factor is also an essential regulator of gene responsible for encoding pro-inflammatory cytokines such as IL-6, IL-12, and MAP-1, the anti-inflammatory drugs targeting NF-kB signaling pathway might hold wide-range therapeutic potential for CCA research (Karin, 2006). Activation-induced cytidine deaminase (AID) belongs to the DNA/RNA editing enzyme family and can be induced by pro-inflammatory cytokines in cholangiocytes. The aberrant production of AID has been previously reported to assist development of CCA via its mutagenic activity (Komori et al., 2008). In addition, studies on CCA-associated patients with a history of chronic inflammation were reported to exhibit abnormally high levels of AID along with germinal center-associated nuclear protein that might be related to DNA damage potentially leading to hepatobiliary malignancy (Chan-On et al., 2009). Over expression of inducible nitric oxide synthase due to prolonged inflammatory response and subsequent inhibition of DNA repair mechanism by its product, nitric oxide, has been reported to promote or assist CCA progression (Jaiswal et al., 2000). In addition, the CCA-associated tumor cells were reported to exhibit aberrant levels of 8-nitroguanine, usually formed as a result of nitrative DNA damage, along with increased formation of DNA lesions (Thanan et al., 2013). Thus, it clearly indicates the critical role played by inflammatory immune response that can potentially lead to CCA.

\section{Pathogens Associated with Cholangiocarcinoma}

Epidemiological and experimental evidences suggest that some helminthic, bacterial and viral pathogen may act as carcinogenic agents in the development of CCA. In particular, patients suffering from infestation of certain types of liver flukes namely, Opisthorchis viverrini and Clonorchis sinensis are presumably more vulnerable to CCA (Boonyanugomol et al., 2012). The O. viverrini infestation is prevalent in parts of Thailand, Cambodia, and Laos correlating with the high incidents of CCA in these countries as compared to other parts of the world (Parkin, 2006; Sripa and Pairojkul, 2008). These parasites survive within bile duct and are known to produce multiple excretory proteins including superoxide dismutase, venom allergen-like proteins, and granulin-like growth factor that can potentially contribute to malignant transformation of cholangiocytes (Young et al., 2014). Likely, the gastrointestinal tract-associated bacterium Helicobacter pylori is considered as a significant risk factor in development of CCA (Kawanishi et al. 2006; Matsumoto et al., 2007; Xiao et al., 2014). It has been suggested that the cag pathogenicity island found in $H$. pylori which were previously known to be involved in pathogenesis of gastric epithelial cells have now been linked to the development of biliary carcinoma (Matsumoto et al., 
2007; Boonyanugomol et al., 2013). Likely, the hepatitis $\mathrm{B}$ virus (HBV) and hepatitis $\mathrm{C}$ virus (HCV) are generally associated with hepatocellular carcinoma; however, these viral infections are now also being considered as an important risk factor for CCA (Zhou et al., 2014; Matsumoto et al., 2014). Clinical studies indicate that patients suffering from CCA are often found to express high seroprevalence of HBV and HCV hepatitis surface antigens indicating a medical history of viral hepatitis $(\mathrm{Qu}$ et al., 2011; Matsumoto et al., 2014). A separate study suggested that the HBV-associated CCA contained distinct clinicopathological features and these patients tend to contain abnormally high levels of $\alpha$-fetoprotein and low levels of serum carbohydrate antigen 19-9 (CA 19-9) as compared to the CCA patients without $\mathrm{HBV}$ infection $(\mathrm{Wu}$ et al., 2013). Hence, even though the detailed mechanism of host-pathogen interaction is barely understood, it is clear that infection by such pathogens can considerably increase the predisposition to development of CCA.

\section{Biomarkers in Cholangiocarcinoma}

Patients with early stages of CCA are often asymphomatic or display non-specific symptoms such as cachexia, abdominal pain, and fatigue (Blechacz et al., 2011). Patients are often diagnosed with CCA only during the advanced stages of carcinoma where surgical procedures become inapplicable for curing the disease (Sriwanitchrak et al., 2011). Absence of effective early diagnostic techniques and limited therapeutic options make it difficult to prevent CCA-associated mortalities. Thus, there is an urgent need for identification of biomarkers to assist effective diagnose of CCA during early stage of the disease. A few serum-derived biomarkers including carcinoembryonic antigen and CA19-9 have shown some promising signs for its application in timely diagnosis of CCA (Patel et al., 2000; Marrero, 2014). However, the markers suffer from low sensitivity and cannot discriminate between malignant and benign form of biliary cholangitis (Sriwanitchrak et al., 2011). In particular, CA19-9 is suggested to have sensitivity of up to $80 \%$ in diagnosing perihilar CCA and distal CCA (Tao et al., 2010). Moreover, in patients suffering from PSC, a combined approach involving measurement of serum CA19-9 level and cytopathological studies of hepatic tissues were used as a strong predictive test for CCA development (Barr et al., 2013). Likely, the cytopathological techniques such as fluorescence in situ hybridization (FISH) in patients with PSC has identified polysomy in chromosome 3 and 7 as a significant riskfactor for increased predisposition to iCCA. Currently, FISH assay are being recommended to PSC patients as a predicting test to access their genetic susceptibility towards CCA genesis (Navaneethan et al., 2013). The cytokeratins $(\mathrm{CK})$ represents an essential group of proteins that contribute to cytoskeletion of various cell types. Over 20 different types of CKs have been identified and CK-19, specifically expressed in bile duct epithelia, is known to be associated with CCA (Stroescu et al., 2006). The CK19 is considered as a biomarker specific to iCCA that can be used to distinguish intrahepatic cholangiocarcinoma from hepatocellular carcinoma (Tao et al., 2010; Marrero, 2014). In addition, high levels of CK-19 were also detected as a prominent sign of liver fluke-related CCA (Thanan et al. 2013). However, for clinical application, this biomarker requires further validation and currently there exists no precise detection protocol to identify and correlate aberrant levels of CK-19 during early stages of iCCA. Separate studies on inflammation-mediated CCA involving stem/progenitor cell markers (CD133 and Oct3/4) suggested that the aberrant expression of CD133 and/or Oct $3 / 4$ correlates with increased formation of DNA lesions in CCA patients (Thanan et al., 2013). Further studies of these proteins in relation to CCA might hold diagnostic potentials for identification and development of biomarkers based on immunological approach. The hepatoma-derived growth factor (HDGF) is a mobility promoting growth factor and is known to be implicated in promoting metastatic activities of several types of tumor. Recently, the HDGF profiling of CCA-associated patients suggested that HDGF might hold diagnostic potentials as a non-invasive biomarker for detecting extrahepatic CCA and also appears to be a suitable prognostic factor after curative resection of extrahepatic CCA (Han et al., 2013). Likely, multiple signaling proteins and associated factors such as facin, Akt, TROP2, and HSP27 are currently being considered for their potential diagnostic use in CCA research (Ruys et al., 2013). In addition to intracellular molecules, study of bile for its chemical composition and quantitative evaluation of proteins including SSP411, pancreatic esterases and amylases have also been investigated for identification of potential biomarkers with promising results (Alvaro 2009; Shen et al., 2012). List of few selected biomarkers used in some CCA-associated research is provided in this article (Table 1). Hence, identification and establishment of a highly sensitive and robust early diagnostic procedure based on such biomarkers would definitely be a major positive step in CCA research.

\section{Anticancer Drug Research on Cholangiocarcinoma}

Plant-derived chemicals have been used throughout the history of medicine for development of various anticancer and antiinflammatory drugs. In this context, multiple researches have been conducted to identify phytochemicals with potential anticancer properties against CCA. Experiments utilizing in-vitro and nude mouse CCA-xenograft models have suggested that purified curcumin from Curcuma longa Linn., crude ethanolic extract from Zingiber officinale Roscoe, and Atractylodes lancea (thung) possess significant antitumor activity against CCA (Plengsuriyakarn et al., 2012 a \& b). In particular, the anticancer effects of A. lancia were comparable to 5-flurouracil and were most effective for reduction of tumor mass, inhibition of lung metastasis and prolongation of survival time in mouse CCA-xenograft models (Plengsuriyakarn et al., 2012a). In addition, a similar research has previously indicated that the crude ethanolic extracts of $A$. lancea have been reported to exhibit selective and strong anti-cancer properties 


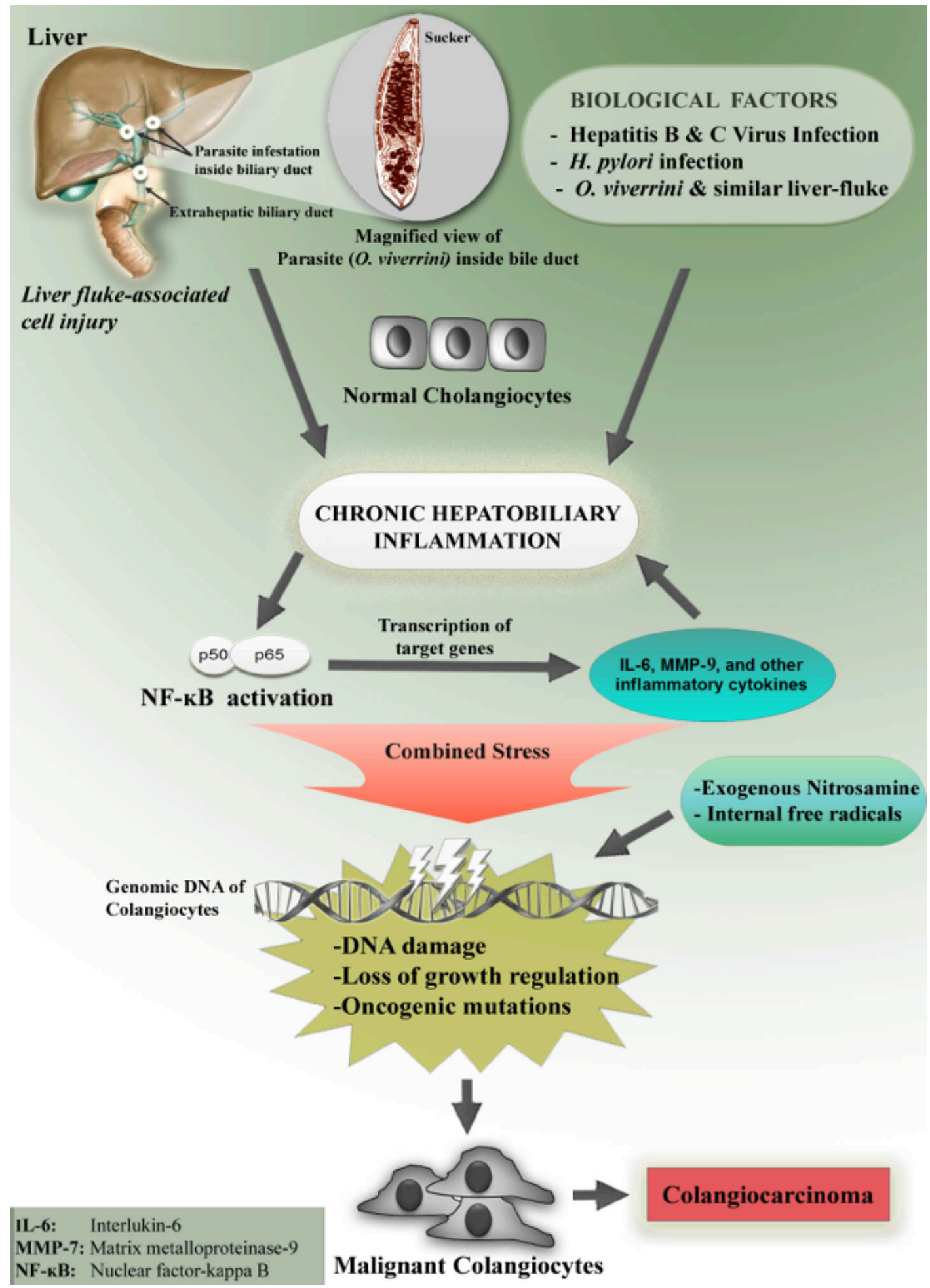

Figure 1. Schematic Overview of Multiple Factors Potentially leading to Cholangiocarcinoma (CCA). Development of CCA involves multiple complex steps with several different factors exerting prolonged stress on hepatic biliary colangiocytes. The cellular stress is often collectively observed as chronic hepatobiliary inflammation with involvement of key inflammatory mediators such as NF-kB and IL-6. The CCA-associated factors may include exogenous nitrosamine, certain liver-fluke infestation, viral hepatitis and Helicobacter pylori infection. Likely, the free radical-mediated genomic DNA damage and genetic susceptibility to oncogenic mutations can also play significant role in malignancy of colangiocytes resulting in CCA

against in vitro cultured CL-6 cells with values for half maximal inhibitory concentration $\left(\mathrm{IC}_{50}\right)$ less than $30 \mathrm{ug} /$ $\mathrm{ml}$ (Mahavorasirikul et al., 2010). Hence, further in-depth mechanistic studies on known pure active components of A. lancea such as: beta-eudesmol, atractylone, and hinesol may hold promising therapeutic potentials for treatment of CCA (Mahavorasirikul et al., 2010; Koonrungsesomboon et al., 2014). The in vitro studies of ethanolic extracts from other Thai medicinal plants including Kaempferia galangal and traditionally used Thai folklore recipe (PraSa-Prao-Yha) have also displayed significant cytotoxic activities against the CCA CL-6 cells (Mahavorasirikul et al., 2010). Recent evidence indicates that capsaicin, an active component found in fruits of plant belonging to 
Table 1. List of a Few Promising Biomarkers as Indicated in Some Studies Associated with Cholangiocarcinoma Research

\begin{tabular}{lll}
\hline Biomarkers & Description & Reference \\
\hline Carbohydrate antigen 19-9 (CA19-9) & Serum marker commonly used in clinical practice & Patel et al. 2000 \\
Carcinoembryonic antigen & Serum marker associated with hepatic cancer & Patel et al. 2000 \\
Cytokeratin-19 (CK-19) & Protein associated with cytoskeleton & Stroescu et al. 2006 \\
Fascin & Actin cross-linking protein monomer & Ruys et al. 2014 \\
Hepatoma-derived growth factor (HDGF) & Factor associated with cell mobility and adhesion \\
CD133 and Oct3/4 & Surface receptor and transcription factor, respectively \\
Mucins & Heavily glycosylated protein produced by epithelial cells $\quad$ Ruys et al. 2014 & Associated with breakdown of extracellular matrix and \\
Metalloproteinase-7 & also promote metastasis of tumor cells & Protein found in serum and also associated with hepatic \\
Spermatogenesis associated 20 (SSP411) & abnormalities & Shen et al. 2012 2014 \\
\hline
\end{tabular}

genus Capsicum, exerts inhibitory effect on cell mobility and invasion of HuCCT1 CCA cells. The anticancer activity is largely attributed to the ability of capsaicin to suppress transcription of matrix metalloproteinase- 9 by interfering with NF-kB signaling pathways (Lee et al., 2014). Similarly, a plant-derived flavonoid, luteolin, is reported to induce apoptosis and arrest cell cycle in human CCA KKU-M156 cells. The luteolin-induced apoptosis was mainly attributed to suppression of anti-apoptotic Bcl2 protein, whereas, the cell cycle progression was largely restrained by inhibiting expression of cyclin A and Cdc25A proteins (Aneknan et al., 2014). In a separate research, administration of melatonin was linked to reduced CCA genesis in hamsters subjected to $O$. viverrini infection and $\mathrm{N}$-nitrosodimethylamine exposure that are known to induce liver injury and CCA (Laothong et al., 2013). In contrast, several commercially available synthetic and semi-synthetic drugs including axitinib, metformin, and celecoxib are also being studied with promising results for their anticancer effects against CCA (Yeh et al., 2013; Takahashi et al., 2014; Ling et al., 2014). Therefore, studies focusing on identification and purification of novel anticancer compounds may hold huge therapeutic potentials and are indispensable part of CCA research.

\section{Conclusions}

CCA is progressively fatal form of hepatobiliary cancer and extremely difficult to diagnose at its early stages. Multiple risk factors and their complex interactions are involved during development of this cancer which remains a topic of extensive research. Epidemiological evidence indicates that the global incidence of CCA is increasing and bears a very dismal prognosis among patients with progressive stage of the disease. Currently, apart from timely detection and immediate surgical resection of the tumor there is no effective treatment strategy to cure CCA. Thus, investigating the role of pathogens-host interaction, host immune response, potential risk factors, and biomarkers associated with CCA is essential in devising effective diagnostic and therapeutic strategies against this disease (Figure 1). In addition, continuous focus should also be given to exploration of natural as well as synthetic anticancer compounds utilizing both in vivo and in vitro experimentation approaches in CCA research. Taken together, all currently available knowledge on this devastating disease must be seriously considered for further research on diagnosis and targeted therapies for treating CCA.

\section{Acknowledgements}

VBM is supported by Chulabhorn International College of Medicine, Thammasat University. KN is supported by The Commission on Higher Education, Ministry of Education of Thailand, Office of Higher Education Commission (NRU Project), and Thammasat University (Excellence Center for Pharmacology and Molecular Biology of Malaria and Cholangiocarcinoma).

\section{References}

Alvaro D (2009). Serum and bile markers for cholangiocarcinoma. Curr Opin Gastroenterol, 25, 279-84

Anderson CD, Pinson CW, Berlin J, Chari RS (2004). Diagnosis and treatment of cholangiocarcinoma. Oncologist, 9, 43-57

Aneknan P, Kukongviriyapan V, Prawan A, et al (2014). Luteolin arrests cell cycling, induces apoptosis and inhibits the JAK/ STAT3 pathway in human cholangiocarcinoma cells. Asian Pac J Cancer Prev, 15, 5071-6

Barr Fritcher EG, Voss JS, Jenkins SM, et al (2013). Primary sclerosing cholangitis with equivocal cytology: fluorescence in situ hybridization and serum CA 19-9 predict risk of malignancy. Cancer Cytopathol, 121, 708-17

Blechacz B, Komuta M, Roskams T, Gores GJ (2011). Clinical diagnosis and staging of cholangiocarcinoma. Nat Rev Gastroenterol Hepatol, 8, 512-22

Boonyanugomol W, Chomvarin C, Hahnvajanawong C, Sripa B, Kaparakis-Liaskos M, Ferrero RL (2013). Helicobacter pylori cag pathogenicity island (cagPAI) involved in bacterial internalization and IL-8 induced responses via NOD1- and MyD88-dependent mechanisms in human biliary epithelial cells. PLoS One, 8,77358

Boonyanugomol W, Chomvarin C, Sripa B, et al (2012). Helicobacter pylori in Thai patients with cholangiocarcinoma and its association with biliary inflammation and proliferation. $H P B$ (Oxford), 14, 177-84

Braconi C, Huang N, Patel T (2010). MicroRNA-dependent regulation of DNA methyltransferase-1 and tumor suppressor gene expression by interleukin-6 in human malignant cholangiocytes. Hepatol, 51, 881-90

Burak K, Angulo P, Pasha TM, et al (2004). Incidence and risk factors for cholangiocarcinoma in primary sclerosing cholangitis. Am J Gastroenterol, 99, 523-6

Chan-On W, Kuwahara K, Kobayashi N, et al (2009). 
Cholangiocarcinomas associated with long-term inflammation express the activation-induced cytidine deaminase and germinal center-associated nuclear protein involved in immunoglobulin V-region diversification. Int $J$ Oncol, 35, 287-95

Fabbri M, Garzon R, Cimmino A, et al (2007). MicroRNA-29 family reverts aberrant methylation in lung cancer by targeting DNA methyltransferases $3 \mathrm{~A}$ and 3B. Proc Natl Acad Sci USA, 104, 15805-10

Han Y, Zhang W, Liu Y (2013). Identification of hepatomaderived growth factor as a potential prognostic and diagnostic marker for extrahepatic cholangiocarcinoma. World J Surg, 37, 2419-27

Jaiswal M, LaRusso NF, Burgart LJ, Gores GJ (2000). Inflammatory cytokines induce DNA damage and inhibit DNA repair in cholangiocarcinoma cells by a nitric oxidedependent mechanism. Cancer Res, 60, 184-90

Johnson C, Han YY, Nathan H, et al (2012). Interleukin-6 and its receptor, key players in hepatobiliary inflammation and cancer. Transl Gastrointest Cancer, 1, 58-70

Karin M. (2006). Nuclear factor- $x \mathrm{~B}$ in cancer development and progression. Nature, 441, 431-6

Kawanishi S, Hiraku Y, Pinlaor S, Ma N (2006). Oxidative and nitrative DNA damage in animals and patients with inflammatory diseases in relation to inflammation-related carcinogenesis. Biol Chem, 387, 365-72

Khan SA, Emadossadaty S, Ladep NG, et al (2012). Rising trends in cholangiocarcinoma: is the ICD classification system misleading us? J Hepatol, 56, 848-54.

Komori J, Marusawa H, Machimoto T, et al (2008). Activationinduced cytidine deaminase links bile duct inflammation to human cholangiocarcinoma. Hepatol, 47, 888-96

Koonrungsesomboon N, Na-Bangchang K, Karbwang J (2014). Therapeutic potential and pharmacological activities of Atractylodes lancea (Thunb.) DC. Asian Pac J Trop Med, 7, 421-8

Laothong U, Pinlaor P, Boonsiri P, et al (2013). Melatonin inhibits cholangiocarcinoma and reduces liver injury in Opisthorchis viverrini-infected and $\mathrm{N}$-nitrosodimethylamine-treated hamsters. J Pineal Res, 55, 257-66

Lee GR, Jang SH, Kim CJ, et al (2014). Capsaicin suppresses the migration of cholangiocarcinoma cells by down-regulating matrix metalloproteinase-9 expression via the AMPK-NFxB signaling pathway. Clin Exp Metastasis, 31, 897-907

Ling S, Feng T, Ke Q, Fan N, et al (2014). Metformin inhibits proliferation and enhances chemosensitivity of intrahepatic cholangiocarcinoma cell lines. Oncol Rep, 31, 2611-8

Mahavorasirikul W, Viyanant V, Chaijaroenkul W, Itharat A, NaBangchang K (2010). Cytotoxic activity of Thai medicinal plants against human cholangiocarcinoma, laryngeal and hepatocarcinoma cells in vitro. BMC Complement Altern Med, 10, 55

Marrero J (2014). Biomarkers in cholangiocarcinoma. Clin Liver Dis, 3, ??

Matsumoto K, Onoyama T, Kawata S, et al (2014). Hepatitis B and $\mathrm{C}$ virus infection is a risk factor for the development of cholangiocarcinoma. Intern Med, 53, 651-4

Matsumoto Y, Marusawa H, Kinoshita K, et al (2007). Helicobacter pylori infection triggers aberrant expression of activation-induced cytidine deaminase in gastric epithelium. Nat Med, 13, 470-6

Nair SS, Bommana A, Bethony JM, et al (2011). The metastasisassociated protein-1 gene encodes a host permissive factor for schistosomiasis, a leading global cause of inflammation and cancer. Hepatol, 54, 285-95

Navaneethan U, Njei B, Venkatesh PG, Vargo JJ, Parsi MA (2013). Fluorescence in situ hybridization for diagnosis of cholangiocarcinoma in primary sclerosing cholangitis: a systematic review and meta-analysis. Gastrointest Endosc, 79, 943-50

Oh SW, Yoon YS, Shin SA (2005). Effects of excess weight on cancer incidences depending on cancer sites and histologic findings among men: Korea national health insurance corporation study. Clin Oncol, 23, 4742-54

Parkin DM (2006). The global health burden of infectionassociated cancers in the year 2002. Int J Cancer, 118, 3030-44

Parkin DM, Ohshima H, Srivatanakul P, Vatanasapt V (1993). Cholangiocarcinoma: epidemiology, mechanisms of carcinogenesis and prevention. Cancer Epidemiol Biomarkers Prev, 2, 537-44.

Patel AH, Harnois DM, Klee GG, LaRusso NF, Gores GJ (2000). The utility of CA 19-9 in the diagnoses of cholangiocarcinoma in patients without primary sclerosing cholangitis. Am J Gastroenterol, 95, 204-7

Plengsuriyakarn T, Viyanant V, Eursitthichai V, et al (2012a). Anticancer activities against cholangiocarcinoma, toxicity and pharmacological activities of Thai medicinal plants in animal models. BMC Complement Altern Med, 12, 12-23

Plengsuriyakarn T, Viyanant V, Eursitthichai V, et al (2012b). Cytotoxicity, toxicity, and anticancer activity of Zingiber officinale Roscoe against cholangiocarcinoma. Asian Pac J Cancer Prev, 13, 4597-606

Prayong P, Mairiang E, Pairojkul C, et al (2014). An interleukin-6 receptor polymorphism is associated with opisthorchiasislinked cholangiocarcinoma risk in Thailand. Asian Pac J Cancer Prev, 15, 5443-7

Qu Z, Cui N, Qin M, Wu X (2012). Epidemiological survey of biomarkers of hepatitis virus in patients with extrahepatic cholangiocarcinomas. Asia Pac J Clin Oncol, 8, 83-7

Razumilava N, Gores GJ (2013). Classification, diagnosis, and management of cholangiocarcinoma. Clin Gastroenterol Hepatol, 11, 13-21

Ruys AT, Groot Koerkamp B, Wiggers JK, et al (2013). Prognostic biomarkers in patients with resected cholangiocarcinoma: a systematic review and meta-analysis. Ann Surg Oncol, 21, 487-500

Shaib Y, El-Serag HB (2004). The epidemiology of cholangiocarcinoma. Semin Liver Dis, 24, 115-25

Shen J, Wang W, Wu J, et al (2012). Comparative proteomic profiling of human bile reveals SSP411 as a novel biomarker of cholangiocarcinoma. PLoS One, 7, 47476

Srikoon P, Kariya R, Kudo E, et al (2013). Diethyldithiocarbamate Suppresses an NF- $x \mathrm{~B}$ dependent metastatic pathway in cholangiocarcinoma cells. Asian Pac J Cancer Prev, 14, 4441-6

Sripa B, Mairiang E, Thinkhamrop B, et al (2009). Advanced periductal fibrosis from infection with the carcinogenic human liver fluke Opisthorchis viverrini correlates with elevated levels of interleukin-6. Hepatol, 50, 1273-81

Sripa B, Pairojkul C (2008). Cholangiocarcinoma: lessons from Thailand. Curr Opin Gastroenterol, 24, 349-56

Sriwanitchrak P, Viyanant V, Chaijaroenkul W, et al (2011). Proteomics analysis and evaluation of biomarkers for detection of cholangiocarcinoma. Asian Pac J Cancer Prev, 12, 1503-10

Stroescu C, Herlea V, Dragnea A, Popescu I (2006). The diagnostic value of cytokeratins and carcinoembryonic antigen immunostaining in differentiating hepatocellular carcinomas from intrahepatic cholangiocarcinomas. $J$ Gastrointest Liver Dis, 15, 9-14

Takahashi H, Ojima H, Shimizu H, Furuse J, Furukawa H, Shibata T (2014). Axitinib (AG-013736), an oral specific VEGFR TKI, shows potential therapeutic utility against 
cholangiocarcinoma. Jpn J Clin Oncol, 44, 570-8

Takeuchi O, Akira S (2010). Pattern recognition receptors and inflammation. Cell, 140, 805-20

Tao LY, Cai L, He XD, Liu W, Qu Q (2010). Comparison of serum tumor markers for inirahepaiic cholangiocarcinoma and hepatocellular carcinoma. Am Surg, 76, 1210-3

Thanan R, Pairojkul C, Pinlaor S, et al (2013). Inflammationrelated DNA damage and expression of CD133 and Oct3/4 in cholangiocarcinoma patients with poor prognosis. Free Rad Biol Med, 65, 1464-72

Tyson GL, El-Serag HB (2011). Risk factors for cholangiocarcinoma. Hepatology, 54, 173-84

Ustundag Y, Bayraktar Y (2008). Cholangiocarcinoma: a compact review of the literature. World J Gastroenterol, 14, 6458-66

Wehbe H, Henson R, Meng F, Mize-Berge J, Patel T (2006). Interleukin-6 contributes to growth in cholangiocarcinoma cells by aberrant promoter methylation and gene expression. Cancer Res, 66, 10517-24

Wu ZF, Yang N, Li DY, Zhang HB, Yang GS (2013). Characteristics of intrahepatic cholangiocarcinoma in patients with hepatitis B virus infection: clinicopathologic study of resected tumours. J Viral Hepat, 20, 306-10

Xiao M, Gao Y, Wang Y (2014). Helicobacter species infection may be associated with cholangiocarcinoma: a metaanalysis. Int J Clin Pract, 68, 262-70

Yao D, Kunam VK, Li X (2014). A review of the clinical diagnosis and therapy of cholangiocarcinoma. $J$ Int Med Res, 42, 3-16

Yeh CN, Chiang KC, Juang HH, et al (2013). Reappraisal of the therapeutic role of celecoxib in cholangiocarcinoma. PLoS One, 8, 69928

Young ND, Nagarajan N, Lin SJ, Korhonen PK et al (2014). The Opisthorchis viverrini genome provides insights into life in the bile duct. Nat Commun, 5, 4378

Zhou HB, Hu JY, Hu HP (2014). Hepatitis B virus infection and intrahepatic cholangiocarcinoma. World J Gastroenterol, 20, 5721-9 